WEAVER, C.A. IIIrd. (1993) Do you need a "flash" to form a flashbulb memory? Journal of Experimental Psychology: General, 122, 39-46.

University of Western Ontario

H. MerskeY

London, Canada

\section{Publication bias and meta-analysis}

SIR: Piccinelli et al (1995) present a meta-analysis examining drug treatment in obsessive-compulsive disorder, which demonstrates the efficacy of antidepressant drugs in treatment.

In the absence of large scale randomised control studies, we believe that such meta-analyses may be a valid method of obtaining useful information by pooling data from several smaller studies. However, the results need to be treated with caution since recent meta-analyses in other spheres of medicine have provided misleading results which have subsequently been refuted by large scale randomised control trials.

A recent investigation (Egger \& Davey Smith, 1995) into one 'false positive' meta-analysis suggested the problem was publication bias in the studies available for pooling; small studies reporting a favourable result in treatment groups are more likely to be published. Publication bias can be demonstrated graphically by a 'funnel plot', which plots clinical effect against sample size. If there is no publication bias, then the plot resembles an inverted funnel, since the results from smaller studies are more widely but still symmetrically distributed than those of larger studies.

Piccinelli et al acknowledge that "Published clinical trials may be biased in favour of significant results, since trials failing to show any treatment difference may be less likely to be published". However, they do not test this possibility and since the sample sizes of the studies included were not presented, the critical reader cannot construct funnel plots from their data.

As it has been shown that such biased data can result in misleading results, we would suggest that sensitivity analyses such as funnel plots be included in all systematic reviews presented in the BJP. The science of systematic review remains an evolving one and if it is to retain its credibility then it should be seen to learn from its mistakes.

EGGER, M. \& DAVEY, G. (1995) Misleading meta-analysis. British Medical Journal, 310, 752-754.

Piccinelli, M., Pini, S., Bellantuono, C., et al (1995) Efficacy of drug treatments in obsessive-compulsive disorder; A metaanalytic review. British Journal of Psychiatry, 166, 424-443.

Leeds General Infirmary

S. GILBODY

A. House

Leeds LSI 3EX

\section{Women's response to adversity}

SIR: In their study of women's adjustment to adversity, Surtees (1995) appears to suggest that the experience of entering a women's refuge is an adverse event, comparable to the loss or threatened loss of a partner through death. They express surprise that women entering a Women's Aid refuge show a reduction in general indicators of distress unlike the other two groups who become more depressed and anxious.

The selection of women entering a refuge as a comparison group seems to completely misunderstand the nature of these womens' experiences. Our recent research (Scott-Gliba et al, 1995) found that women in refuges have typically experienced years of violence, abuse and degradation before they take the decision to leave home. Compared with women who were not in violent relationships, they had high levels of depression, anxiety and post traumatic symptoms which decreased over time, when they were separated from their violent partner. For these women the traumatic event is not the loss of home or financial stability, but the terror that precedes it, to which the women have had to make complex behavioural and cognitive adjustments in order to survive. Although leaving a battering partner is, in itself, not without risks (violence typically escalates when faced with the prospect of abandonment) and may involve considerable material hardship, nevertheless the removal of constant threat for these women is beneficial for their emotional and mental well-being, restores their sense of self-respect and dignity and allows them to re-establish some control over their lives.

SCott-GlibA, E., Minne, C. \& Mezey, G.C. (1995) The psychological, behavioural and emotional impact of surviving an abusive relationship. Journal of Forensic Psychiatry (in press).

SURTEES, P.G. (1995) In the shadow of adversity: the evolution and resolution of anxiety and depressive disorder. British Journal of Psychiatry. 166, 583-594.

St George's Hospital Medical School

G.C. Mezey London SW17 ORE

\section{Structured abstracts}

SIR: Simpson \& Baldwin (1995) describe a 71-yearold man who developed acute obsessive-compulsive disorder after a right parietal infarct. SPECT scanning revealed, in addition to the infarct, a diminution of cerebral blood flow in the right basal ganglia and temporal areas. The patient's clinical state improved on a combination of clomipramine and cognitive-behavioural psychotherapy. 